\title{
Deriving solar transient characteristics from single spacecraft STEREO/HI elongation variations: a theoretical assessment of the technique
}

\author{
A. O. Williams ${ }^{1}$, J. A. Davies ${ }^{2}$, S. E. Milan ${ }^{1}$, A. P. Rouillard ${ }^{2,3}$, C. J. Davis ${ }^{2}$, C. H. Perry ${ }^{2}$, and R. A. Harrison ${ }^{2}$ \\ ${ }^{1}$ Department of Physics and Astronomy, University of Leicester, UK \\ ${ }^{2}$ Space Science and Technology Department, Rutherford Appleton Laboratory, UK \\ ${ }^{3}$ Department of Physics and Astronomy, University of Southampton, UK
}

Received: 31 July 2009 - Revised: 21 October 2009 - Accepted: 17 November 2009 - Published: 1 December 2009

\begin{abstract}
Recently, a technique has been developed whereby the radial velocity, $V_{r}$, and longitude direction, $\beta$, of propagation of an outward-moving solar transient, such as a Coronal Mass Ejection (CME), can be estimated from its track in a time-elongation map produced using Heliospheric Imager (HI) observations from a single STEREO spacecraft. The method employed, which takes advantage of an artefact of projective geometry, is based on the evaluation of the best fit of the time-elongation profile of the transient, extracted from a time-elongation map, to a set of theoretical functions corresponding to known combinations of radial velocity and direction; here we present an initial theoretical assessment of the efficacy of this technique. As the method relies on the manual selection of points along the time-elongation profile, an assessment of the accuracy with which this is feasible, is initially made. The work then presented assesses theoretically this method of recovering the velocity and propagation direction of solar transients from their time-elongation profiles using a Monte-Carlo simulation approach. In particular, we assess the range of elongations over which it is necessary to make observations in order to accurately recover these parameters. Results of the Monte-Carlo simulations suggest that it is sufficient to track a solar transient out to around $40^{\circ}$ elongation to provide accurate estimates of its associated radial velocity and direction; the accuracy to which these parameters can be estimated for a transient tracked over a particular elongation extent is, however, sensitive to its velocity and direction relative to the Sun-Spacecraft line. These initial results suggest that this technique based on single spacecraft STEREO/HI observations could prove extremely useful
\end{abstract}

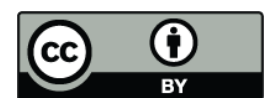

Correspondence to: A. O. Williams (aow2@ion.le.ac.uk) in terms of providing an early warning of a CME impact on the near-Earth environment.

Keywords. Solar physics, astrophysics,and astronomy (Flares and mass ejections; Instruments and techniques)

\section{Introduction}

In their comprehensive analysis of Coronal Mass Ejections (CMEs) observed using the LASCO coronagraphs on the SOHO spacecraft (Brueckner et al., 1995), Sheeley et al. (1999) introduced the concept that the time-elongation profile of CMEs would exhibit an apparent acceleration or deceleration imposed by the observing geometry (elongation being the angle from the line to Sun-centre to the lineof-sight). For the LASCO coronagraphs, the images from which extend to an outer limit of only $8^{\circ}$ elongation, the authors assessed that this effect would be inconsequential. However, with the advent of heliospheric imaging out to far greater elongations - both by the Solar Mass Ejection Imager (SMEI) on the Coriolis spacecraft (Eyles et al., 2003) and more recently, the Heliospheric Imagers (HI) on the pair of NASA Solar TErrestrial RElations Observatory (STEREO) spacecraft (Eyles et al., 2009) - this artefact of the observing geometry is significant and is, indeed, proving to be extremely useful (e.g. Rouillard et al., 2008, 2009a, b, c, d; Sheeley et al., 2008a, b).

For any given solar transient viewed by an observer situated at a radial distance $r_{A}$, from the sun, the shape of its time-elongation profile (the variation of elongation, $\alpha$, as a function of time, $t$ ) depends upon its radial velocity, $V_{r}$, and

Published by Copernicus Publications on behalf of the European Geosciences Union. 


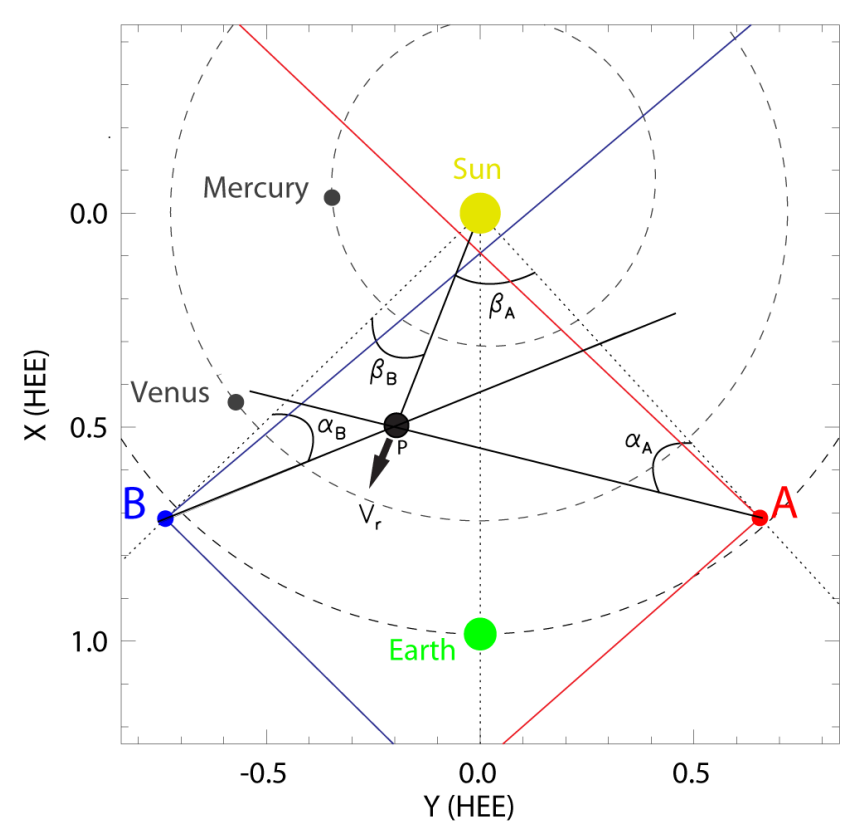

Fig. 1. The locations of STEREO A(head: red) and B(ehind: blue) in the ecliptic plane on 1 January 2009, illustrating the extent of the $\mathrm{HI}$ field of view on each spacecraft. The elongation of $\mathrm{P}$, which corresponds to a solar transient moving with velocity $V_{r}$. at an angle $\beta_{A}\left(\beta_{B}\right)$ with respect to STEREO A (B), is $\alpha_{A}$ and $\alpha_{B}$ for spacecraft $\mathrm{A}$ and $\mathrm{B}$, respectively.

the angle between its propagation path and the Sun-observer line, which we call $\beta$ (Rouillard et al., 2008):

$\alpha(t)=\arctan \left[\frac{V_{r} t \sin \beta}{r_{A}(t)-V_{r} t \cos \beta}\right]$,

Although $r_{A}$ is quoted in Eq. (1) as a time-dependant term, the radial distance of either STEREO spacecraft varies little on the timescale of days (i.e. for the duration over which a solar transient can be tracked). Implicit in the derivation of Eq. (1) are the assumptions that $V_{r}$ and $\beta$ are constant and that the transient has limited spatial dimensions, i.e. that it is a point. As will be discussed later, these assumptions are not necessarily always valid - transients can accelerate and decelerate, and they can be large in spatial extent so it is worth bearing mind that Eq. (1) works best when applied to narrow CME's travelling at speeds close to the solar wind speed. Finally the Thomson Sphere can also affect the visibility of events in the J-maps, as once events move outside of the Thomson Sphere the intensity starts to fall off more rapidly.

For an observer close to the ecliptic plane (as is the case for both the Coriolis and STEREO spacecraft) viewing a solar transient passage along the ecliptic, $\beta$ corresponds to the longitude difference between the observer and transient event in an ecliptic coordinate system. This is illustrated in Fig. 1, which shows the location of the two STEREO spacecraft in the ecliptic plane (X-Y plane in Heliospheric Earth Ecliptic coordinates, HEE) on 1 January 2009; the locations of the Sun, Earth and other inner planets are also shown. Angles $\alpha_{A}$ and $\alpha_{B}$ in Fig. 1 correspond to the elongation of a point $\mathrm{P}$ in the inner heliosphere, as viewed from STEREO A and B, respectively. P can be considered to be the location, at time $t$, of a solar transient propagating anti-sunward with a radial velocity $V_{r}$ at longitudes $\beta_{A}$ and $\beta_{B}$ with respect to STEREO A and $\mathrm{B}$.

The elongation variation of a solar transient can be analysed to provide unique estimates of its associated $V_{r}$ and $\beta$ given a long enough time range of observations. Such analysis has been performed using STEREO HI observations of transients entrained in Corotating Interaction Regions (CIRs) by Rouillard et al. (2008, 2009a, d) and of CMEs by Davies et al. (2009), Davis et al. (2009), Savani et al. (2009), Baker et al. (2009), and Rouillard et al. (2009b). A number of these authors have validated the accuracy of the technique by comparison with in situ measurements by various spacecraft (e.g. including Venus Express or ACE), with ground based observations (e.g. magnetometers), and with solar surface signatures (e.g. by using precise flare locations). The analysis as performed by those authors requires the manual selection of points along the elongation-time profile of an individual transient in a time-elongation map (commonly referred to as a Jmap, Sheeley et al., 1999). The selected points are then compared to a suite of elongation variations generated theoretically using Eq. (1) for all physically realistic combinations of $V_{r}$ and $\beta$, in order to ascertain which combination defines the theoretical curve that most closely fits the selected points.

The work presented here aims to assess theoretically this method of recovering the velocity and trajectory of solar transients from their elongation variations using a Monte Carlo simulation approach. In particular, we aim to assess the range of elongations over which it is necessary to make observations in order to accurately recover $V_{r}$ and $\beta$. In doing this we are then able to comment on the usefulness of this method based on single spacecraft STEREO/HI observations in predicting Earth-bound space weather events.

\section{Instrumentation}

The pair of STEREO spacecraft were launched in October 2006 into heliocentric orbits of approximately 1 AU radius. STEREO-A (ahead) leads the Earth in its orbit, while STEREO-B (behind) trails the Earth; the angular separation between each spacecraft and the Earth increases by approximately $22.5^{\circ}$ every year. In addition to a complement of in-situ instrumentation, each STEREO spacecraft carries the SECCHI imaging suite (Howard et al., 2008) comprising an Extreme Ultraviolet Imager (EUVI), two Sun-centred coronagraphs (COR1 and COR2), and the Heliospheric Imagers (HI) that provide the focus for the current study. The HI instrument (Eyles et al., 2009) consists of two visible-light cameras, HI-1 and HI-2. The HI-1 camera has a $20 \times 20^{\circ}$ 
field-of-view with its bore sight directed at $14^{\circ}$ elongation; the bore sight of the $70^{\circ}$ wide HI-2 field-of-view is aligned at $53.7^{\circ}$. The full angular extent of the HI field of view in the ecliptic plane for each STEREO spacecraft is shown in Fig. 1. The upper panel of Fig. 2 shows the angular extent and relative locations of the HI-1 and HI-2 fields of view by presenting a pair of near-simultaneous running difference images from the STEREO A spacecraft on 12 July 2007. The images are plotted in terms of elongation angle along the central row of the combined HI field-of-view (horizontal dashed line) and elevation angle perpendicular to that line. The upper panel also shows elongation contours indicating how the elongation varies with elevation. During the earliest stages of the STEREO mission the bore sights of the two HI instruments were not well aligned with the ecliptic plane, but by the time of the observations used in this paper both instruments were aligned to within $1^{\circ}$ of the ecliptic plane. The horizontal dashed line in the image therefore corresponds closely to the ecliptic plane. The HI-1 instrument produces an image every $40 \mathrm{~min}$, while for the HI-2 instrument the interval between images is $120 \mathrm{~min}$.

\section{Observations}

The technique of creating time-height maps (J-maps) of coronal data, initially developed by Sheeley et al. (1999) to aid the characterisation of CMEs observed by the LASCO coronagraphs on SOHO, has more recently been applied to observations from the STEREO HI instruments, although with height being substituted by the more appropriate elongation parameter. In essence, the J-mapping technique, as has generally been applied to the $\mathrm{HI}$ data, involves plotting the intensity along a fixed solar radial (usually the ecliptic) from a series of running difference HI-1 and HI-2 images as a function of time on the $\mathrm{X}$-axis and elongation on the Y-axis.

The upper panel of Fig. 2 combines HI-1 and HI-2 running difference images from 12 July 2007, during the passage of a CME through the HI field-of-view of the STEREO A spacecraft. HI observations of this CME, the launch of which was associated with NOAA Active Region 10963, have previously been presented by Davies et al. (2009). As noted above, the extraction of the time-elongation variation for a transient has been done manually by the authors applying this technique.This CME exhibits a typical 3-part morphology, with a hierarchy of overlying loops followed by a density cavity and a subsequent dense, and in this case Vshaped, core. In the difference images, light grey/white areas reveal areas in which the intensity is increased relative to the previous image (corresponding to increased electron density) whereas dark grey/black areas show areas of reduced intensity (reduced density). Note that the visibility of a solar transient will not only be altered by changes in its density but also by its proximity to the Thomson sphere, as will be discussed in more detail later. A mask is applied to the HI-2 image

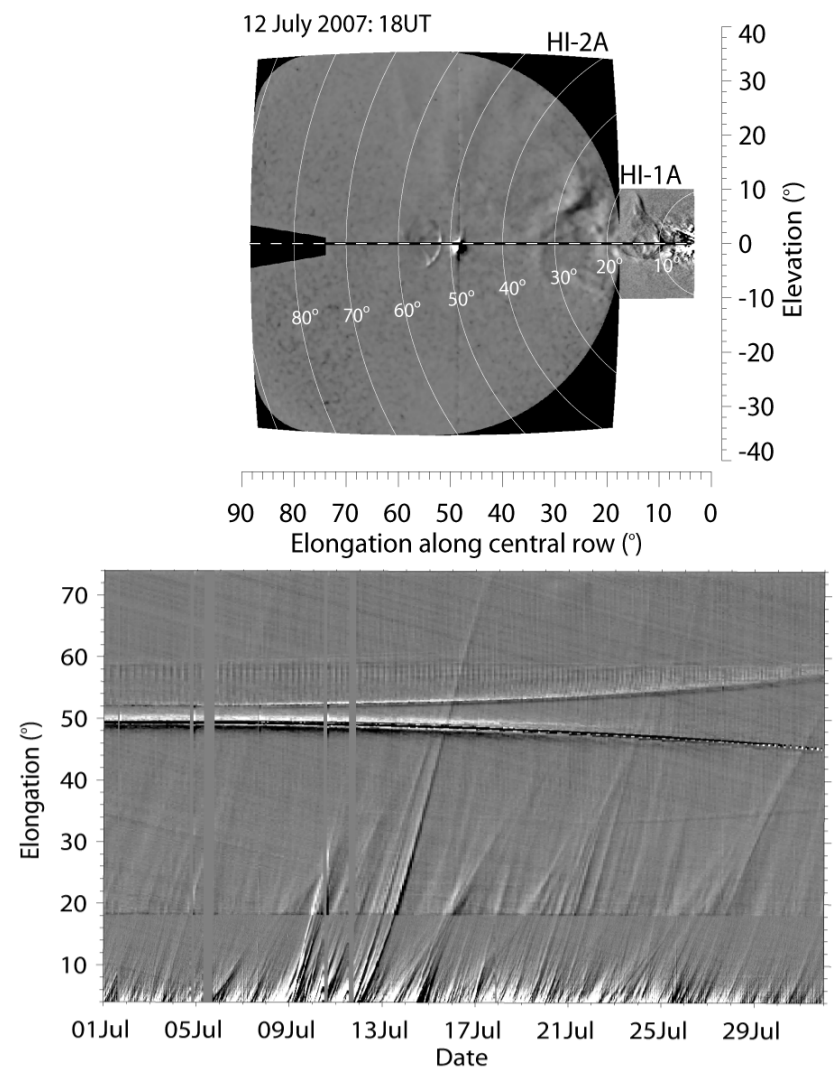

Fig. 2. The upper panel presents combined differenced images from the HI-1 and HI-2 instruments aboard the STEREO A spacecraft from 18:00 UT on 12 July 2007. The dashed line corresponds to the ecliptic, along which the J-map shown in the lower panel is produced and the curved lines show contours of constant elongation. The J-map extends over July 2007 and gaps due to missing images are filled in grey. The tracks of many antisunward-moving solar transients can be seen in the J-map.

to conceal areas of the field-of-view obscured by the straylight baffle system and the Earth occulter. The lower panel of Fig. 2 illustrates a time-elongation (J-) map extending over the entire of July 2007, the month encompassing this CME, following the method described by Davies et al. (2009). The J-map presented here is derived from HI- 1 and HI-2 running difference observations along the centre of the combined $\mathrm{HI}$ field-of-view on STEREO A, which for this interval of nominal instrument operation corresponded closely to the ecliptic. The elongation range of the J-map extends from $4^{\circ}$, the sunward edge of the in-ecliptic portion of the HI-1 field-of-view, to $74^{\circ}$, a limit placed on observations of the central part of the viewing area by the Earth occulter.

The J-map reveals many inclined tracks, corresponding to antisunward-moving solar transients, which are visible out to different elongations. The clearest track, which enters the HI-1 field-of-view early on 12 July and can be observed propagating antisunward until it finally becomes obscured by the Earth occulter on 17 July, corresponds to the dense 


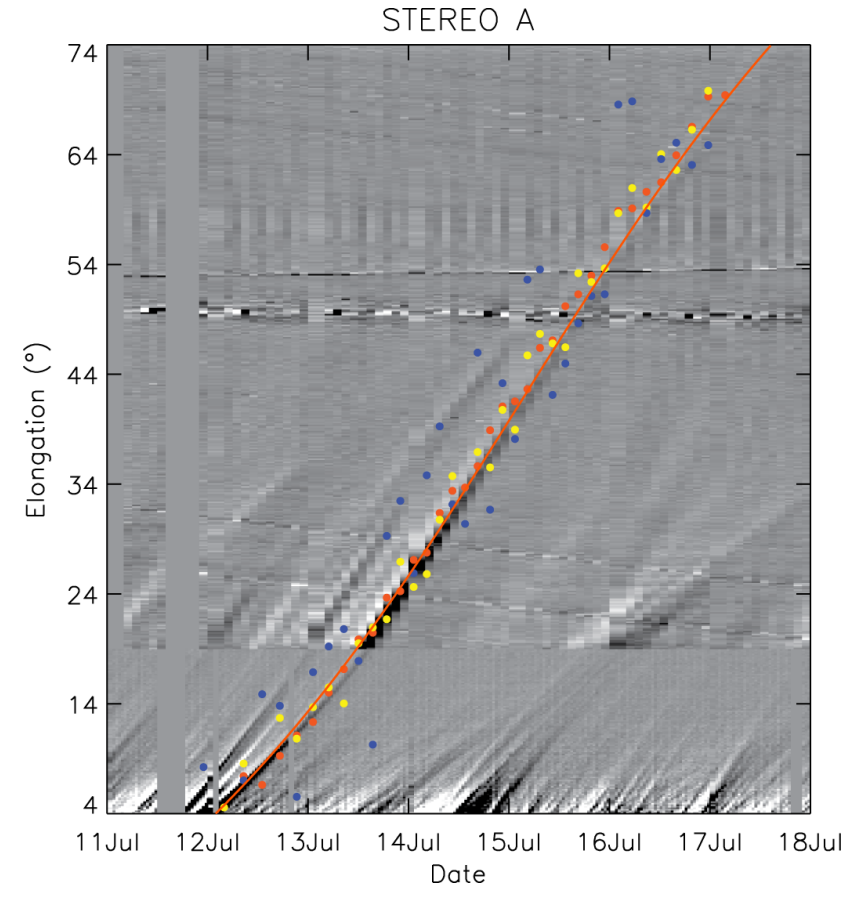

Fig. 3. A close up view of one of the features seen in Fig. 2, with three sets of points overlaid simulating three levels of accuracy with which a person can track the feature. The orange, yellow and blue dots mark sets of normally distributed points with standard deviations of 1,2 and $5^{\circ}$, respectively, around those points determined theoretically for a solar transient with $V_{r}=320 \mathrm{~km} \mathrm{~s}^{-1}$ and $\beta=48^{\circ}$.

core of the CME imaged in the top panel of Fig. 2. Note the transition between light and dark within the core trace results from use of difference images. Preceding this track are fainter tracks corresponding to the overlying loops of the CME front.

As stated previously, from the elongation variation of a given solar transient, extracted from such a J-map, it is possible to estimate its radial velocity, $\mathrm{Vr}$, and the angle at which it is propagating relative to the Sun-spacecraft line, $\beta$. Following the method of Rouillard et al. (2008, 2009d), Davies et al. (2009) estimated the CME core to be propagating with a radial velocity of $320 \mathrm{~km} \mathrm{~s}^{-1}$ at a longitude relative to the Sun-STEREO A line of $48^{\circ}$ in the ecliptic plane. These values were validated by in-situ and solar surface observations, the latter of which revealed a launch time of 04:00 UT on the 11 July 2007 in the vicinity of active region 10963. CME activity was also recorded in the STEREO inner and outer coronagraphs and SOHO/LASCO coronagraphs.

The accuracy of the velocity and direction estimates retrieved from fitting the elongation variation is clearly going to be dependent on the accuracy with which the user is able to follow a specific track. This is assessed in Fig. 3, which reproduces that portion of the J-map presented in Fig. 2, lower panel, extending from 11 to 17 July 2007 and covering the CME passage throughout the STEREO-A HI field of view.
Using the CME velocity and longitude quoted by Davies et al. (2009) for the CME core, $V_{r}=320 \mathrm{~km} \mathrm{~s}^{-1}$ and $\beta=48^{\circ}$, its "ideal" elongation variation is calculated from Eq. (1), from an elongation of $4^{\circ}$ out to an elongation of $74^{\circ}$ with an elongation resolution of $2^{\circ}$; this elongation range reflects the maximum extent to which outward propagating solar transients can be tracked through the HI viewing area in the ecliptic plane. Subsequently, three levels of random noise are added onto this ideal elongation variation, normal distributions with standard deviations of 1,2 and $5^{\circ}$, to simulate three different degrees of accuracy with which the user could conceivably trace the ideal curve. These three sets of points are over plotted onto the CME core track in the J-map as orange, green and blue dots marking the sets of points with 1,2 and $5^{\circ}$, respectively, from the ideal elongation variation of the CME core. By overlaying these three sets of points onto the J-map, it can be seen that selecting points along this transient track with a standard deviation of $2^{\circ}$, and probably even $1^{\circ}$, from the perfect curve, shown in red, is likely to be realistically achievable. It is possible that in the elongation ranges covered by the HI-1 instrument it would be realistic to assume a standard deviation of less than $1^{\circ}$ due to the increased resolution compared to the HI-2 images. In the elongation ranges covered by HI-2 it is generally the case that transient events drop off in brightness with increasing elongation. Hence it would be realistic to assume an increasing error with increasing elongation with a maximum error of $2^{\circ}$ at larger elongations being a realistic target. Clearly the blue points representing a $5^{\circ}$ error would be completely unrealistic for any event quantified by this method.

\section{Analysis of the 12 July 2007 CME}

Clearly the choice of points a user makes in selecting a transient will have a significant effect on the derived parameters $V_{r}$ and $\beta$. As discussed previously, in order to retrieve the values of $V_{r}$ and $\beta$ from the elongation variation of a given solar transient, the manually selected points along its track in a J-plot are compared to a suite of elongation variations generated theoretically using Eq. (1) for all physically realistic combinations of $V_{r}$ and $\beta$. As discussed by Rouillard et al. (2009a), the best fit is obtained by evaluating $\sigma$, the standard deviation of the residuals between the observed elongation variation, $\alpha^{\prime}(t)$ and a given theoretical elongation variation, $\alpha(t)$ derived from Eq. (1) for a particular combination of $V_{r}$ and $\beta$.

$\sigma=\sqrt{\frac{1}{N} \sum_{i=1}^{N}\left\{\alpha^{\prime}\left(t_{i}\right)-\alpha\left(t_{i}\right)\right\}^{2}}$

The combination of $V_{r}$ and $\beta$ that correspond to the theoretical elongation that gives rise to the best fit defined by the minimum in this parameter $\sigma$ are then assigned to the transient. 
In order to demonstrate more explicitly the effect on the goodness of fit of the level of accuracy with which the user can track this transient, Fig. 4 plots $\sigma$ (which we subsequently call the fit error) obtained when fitting each of the three "observed" elongation variations over plotted on the Jmap in Fig. 3, contoured as a function of $V_{r}$ and $\beta$. The topleft, bottom-left and top-right panels of the figure correspond to fitting the elongation variations with random errors of $1^{\circ}$, $2^{\circ}$ and $5^{\circ}$ standard deviation, respectively. In each case the fit error is evaluated by comparing the observed elongation variations to theoretical elongation variations computed over a range of $V_{r}$ values of 0 to $1000 \mathrm{~km} / \mathrm{s}$ (with a resolution of $1 \mathrm{~km} \mathrm{~s}^{-1}$; y-axis) and a range of $\beta$ values of 0 to $100^{\circ}$ (with a $1^{\circ}$ resolution; $\mathrm{x}$-axis). A white star in each panel mark the combination of $V_{r}$ and $\beta, 320 \mathrm{~km} \mathrm{~s}^{-1}$ and $48^{\circ}$ respectively, from which the three observed elongation variations are subsequently derived. The white diamond marks the $V_{r} / \beta$ combination which is calculated to produce the best fit to the observed elongation variation in each case, by virtue of corresponding to the minimum fit error. Each panel also has a white contour line overlaid which marks the region bounded by values of $\sigma$ equal to twice its minimum value.

It is clear from Fig. 4 that the parameters corresponding to the best fit (the diamond) are closest to the input parameters (marked with a cross) when the user fit to the transient track is performed most accurately (top-left panel). This results from the much more tightly constrained minimum in the fit error, $\sigma$, as is evident by comparing the contours defined by the 2 times $\sigma$ level. Also, as would be anticipated, the minimum value of the fit error is higher for the tracks with larger random error. For the case of the most poorly defined track (with its points having a random error of $5^{\circ}$ standard deviation; top-right panel) the fit error field is highly uniform, so much so that this contour encompasses $V_{r}$ values from $300 \mathrm{~km} \mathrm{~s}^{-1}$ to $600 \mathrm{~km} \mathrm{~s}^{-1}$ and $\beta$ values from $20^{\circ}$ to $100^{\circ}$. It is interesting to note that the form of the fit error field is such that, for this combination of initial parameters at least, it appears that one is likely to be able to determine $V_{r}$ much more accurately than $\beta$. Obviously, as the noise added is random, with only its standard deviation specified, different runs would produce somewhat different results; we will return to this theme later when we adopt a Monte Carlo simulation scheme to compare the accuracy of fitting different extents of the elongation variation. While this $5^{\circ}$ standard deviation is shown mostly for comparison, it emphasises the need for the users to take care in selecting points for fitting.

Based on Fig. 3, we decide that it is realistic to assume that a user is capable of selecting a set of points along a typical transient track with a $2^{\circ}$ standard deviation in the worst case at elongations greater than $44^{\circ}$, a $1^{\circ}$ standard deviation between $24^{\circ}$ and $44^{\circ}$, and a $0.5^{\circ}$ standard deviation in the range of the HI- 1 instrument of $4^{\circ}$ to $24^{\circ}$ elongation. We examine the effect on the accuracy of the best fit parameters of the extent over which the solar transient can be tracked by the HI instruments. Differences between the points selected by
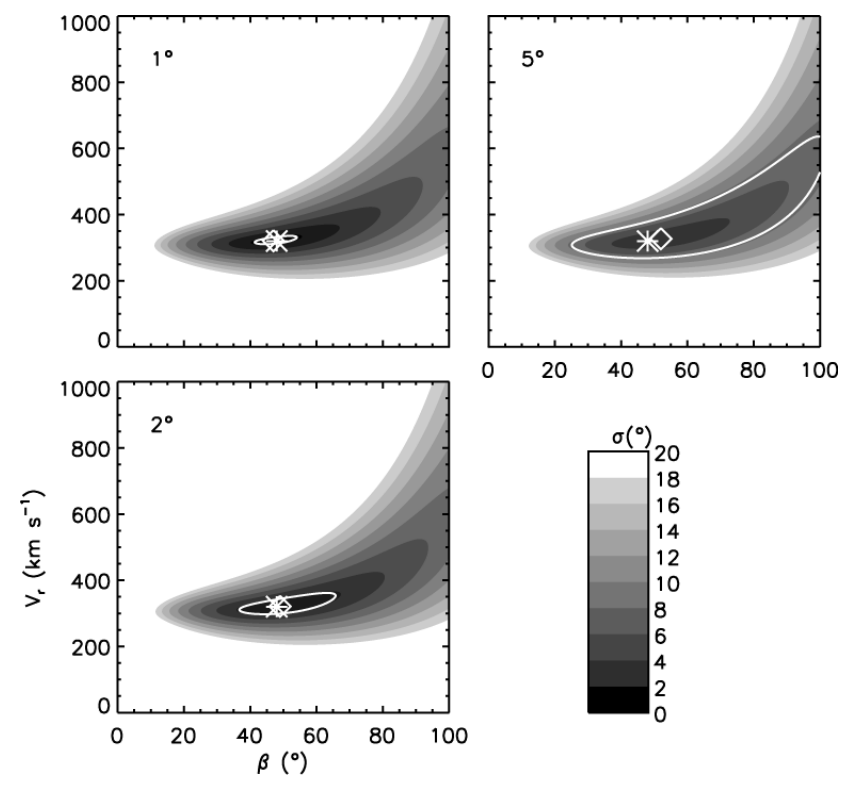

Fig. 4. Contour plots of the fit errors obtained when fitting elongation variations corresponding to a track defined by $V_{r}=320 \mathrm{~km} \mathrm{~s}^{-1}$ and $\beta=48^{\circ}$ onto which three levels of random error have been added (with 1,2 and $5^{\circ}$ standard deviation - top left, bottom left and top right panels, respectively). Darker shades illustrate regions of lower fit errors, lighter shades reveal larger fit errors. The asterisk on each panel marks the input values, while the diamond marks the best fit values. The white contours here indicate a level equal to twice the minimum $\sigma$.

different observers (and indeed a single observer doing multiple fits of the same transient) could also result in a different value of $V_{r}$ and $\beta$ for a transient. The results presented here provide observers with a method of determining the quantitative impact this will have on the parameters $V_{r}$ and $\beta$. J-plots such as those shown in Fig. 2 (lower panel) and Fig. 3, reveal that only a minority of transients can be tracked over the full range of elongations viewed by $\mathrm{HI}, 4^{\circ}$ to $74^{\circ}$, in the ecliptic plane. As well as the precision with which the user is able to follow the transient signature in a J-plot, it is suggested that the elongation extent over which it can be tracked will also affect the accuracy of retrieving the parameters from the observed elongation variation; this we assess now.

Figure 5 presents contour plots of the errors in fitting, similar to those presented in Fig. 4, but where the elongation profile is fitted over decreasing ranges of elongation $4^{\circ}$ to $74^{\circ}$, $4^{\circ}$ to $64^{\circ}, 4^{\circ}$ to $54^{\circ}$ and $4^{\circ}$ to $44^{\circ}$ (a-d), $4^{\circ}$ to $34^{\circ}, 4^{\circ}$ to $24^{\circ}$ and $4^{\circ}$ to $14^{\circ}(\mathrm{e}-\mathrm{g})$; this simulates the effect of this technique of tracking solar transients that are only visible in the HI images to different points in the field of view. As in Fig. 4 the asterisk and diamond mark the initial input and best fit values, respectively, and a white contour marks the boundary of twice the minimum value of $\sigma$. The top-left panel, showing the error map derived from fitting the elongation variation of a solar transient which can be detected over the full extent 

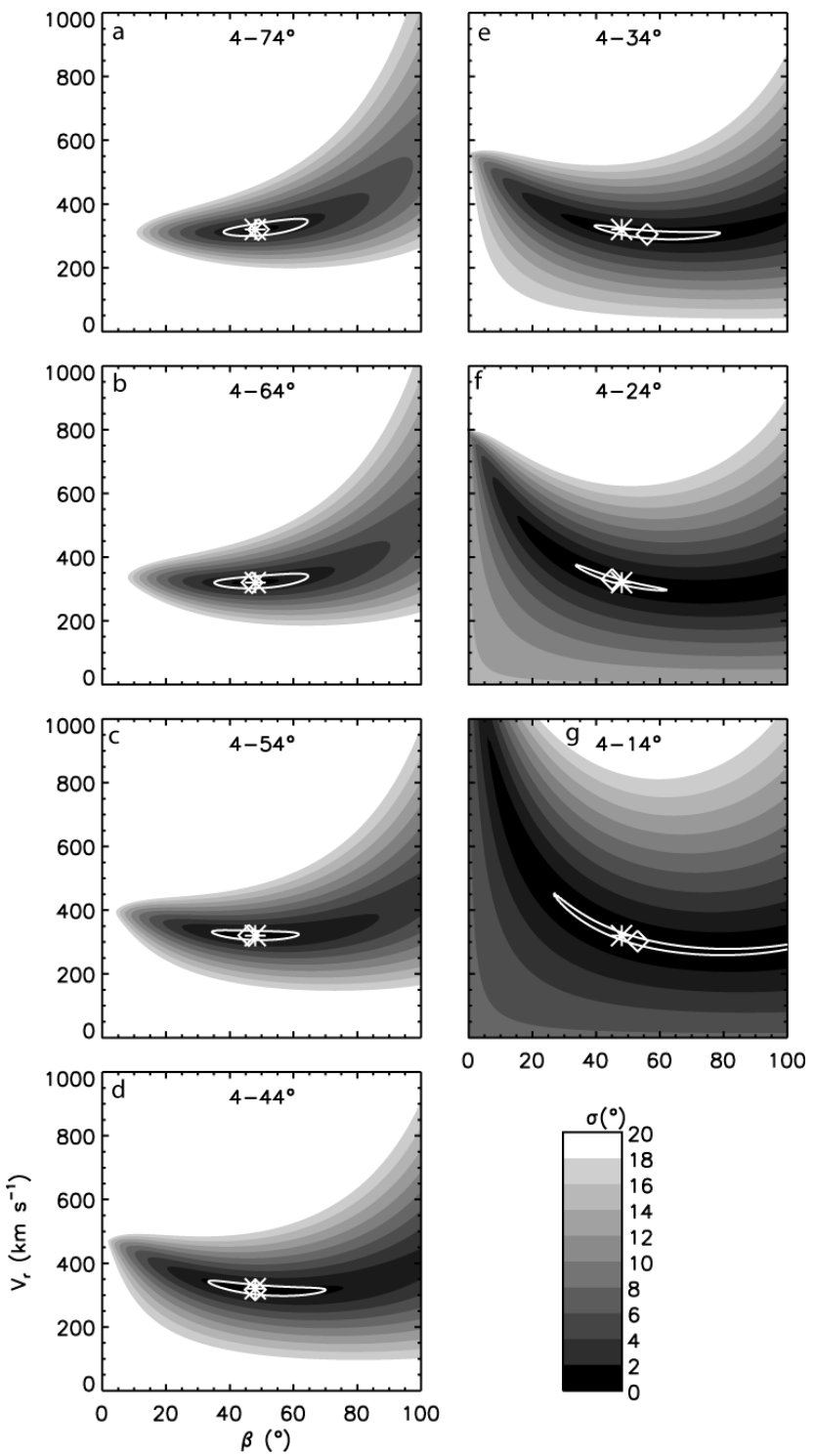

Fig. 5. Contour plots of the fit errors obtained when fitting decreasing extents of the elongation variation corresponding to a track defined by $V_{r}=320 \mathrm{~km} \mathrm{~s}^{-1}$ and $\beta=48^{\circ}$ onto which a random error has been added as described in the text. In each panel the Asterisk marks the input values, and the diamond marks the best fit values. Each panel corresponds to the elongation range given. The white line marks a contour equal to twice the minimum fit error.

of both HI cameras, from $4^{\circ}$ to $74^{\circ}$, will be similar to the bottom left panel of Fig. 4; differences will exist though due to the smaller errors used at elongations less than $44^{\circ}$; the main notable difference is a better certainty in the fit from the $2 \sigma_{\min }$ level in Fig. 5. The $2 \sigma_{\min }$ level here corresponds to a certainty of $V_{r}$ and $\beta$ of $95 \%$. The panels showing fit errors associated with fitting an event seen further than approximately halfway across the HI 2 field of view, panels (b-d), potentially give a very good fit. It should be noted that the range of values that form the smallest mean error is slightly larger than when using the full extent of the cameras and this range increases as the visible extent decreases. Panels $(\mathrm{e}-\mathrm{g})$ use a smaller extent covering the HI 1 camera and sunward portion of the HI 2 camera. The result of fitting over shorter elongation extents is clear: rather than a band running across a range of $\beta \mathrm{s}$ and a narrow range of velocities, there is a broader spread that runs from high $V_{r}$ /small $\beta$ values to large $\beta / V_{r}$, again as shown by the overlaid white contour lines. The best fit is again marked with a diamond and, in general, the panels (a-e) which use the HI 2 camera have a best fit which is very close to the perfect fit.

\section{Monte-Carlo simulations}

We have until now looked at the results of fitting an observed elongation variation defined by one pair of input parameters, with a set of normally distributed errors dependent on elongation. As the addition of errors invokes a random number generating technique, it is clear that it can be done many times, with the resultant profile being fitted for each. Thus we use a Monte Carlo method to determine the mean absolute errors between the retrieved $V_{r}$ and $\beta$ and the initial values over a range of simulated events. Here, instead of comparing the observed elongation values with the theoretical elongation values, the best fit $V_{r}$ and $\beta$ are compared to the input value. Equation (2) is used to find the best fit and this is done 1000 times for each input combination. The mean absolute error between the best fit values of $V_{r}$ and $\beta$ and the input values (for each combination of the latter) is stored. This is done for a range of input $V_{r}$ values from $100 \mathrm{~km} \mathrm{~s}^{-1}$ to $900 \mathrm{~km} \mathrm{~s}^{-1}$ in steps of $50 \mathrm{~km} \mathrm{~s}^{-1}$ and a range of $\beta$ values of $10^{\circ}$ to $90^{\circ}$ in $5^{\circ}$ steps. To simplify matters a $2^{\circ}$ standard deviation error is applied to the entire elongation range and the analysis is applied over the same elongation extents as for Fig. 5. The mean absolute error in $V_{r}$, contoured as a function of $V_{r}$ and $\beta$ is shown in Fig. 6. Similarly the mean absolute error in $\beta$ is shown in Fig. 7.

Figure 6 shows in descending order the error in fitting of $V_{r}$ as a function of the input $V_{r}$ and $\beta$ for fits to elongations of $4^{\circ}$ to $74^{\circ}$ (a), $4^{\circ}$ to $64^{\circ}$ (b), $4^{\circ}$ to $54^{\circ}$ (c), $4^{\circ}$ to $44^{\circ}$ (d), $4^{\circ}$ to $34^{\circ}(\mathrm{e}), 4^{\circ}$ to $24^{\circ}$ (f) and $4^{\circ}$ to $14^{\circ}(\mathrm{g})$. Note that the colour scale on each of the panels is different, with the scale increasing as the range of elongations used in the fit decreases; this can be seen from the overlaid contours. Looking at Fig. 6 as a whole it is interesting to note that the value of $\beta$ with the smallest error in $V_{r}$ increases as the elongation range over which the event is fitted decreases. Figure 6 appears to show that in all cases that the error in $V_{r}$, for any given $\beta$, decreases with decreasing $V_{r}$. However, Fig. 6 presents absolute errors and if we consider percentage errors, there is no variation with $V_{r}$ for any given $\beta$, only with changing $\beta$ does the error change to any appreciable degree. Figure 6 also shows two error populations present, one at high values of $\beta, 65^{\circ}$ 

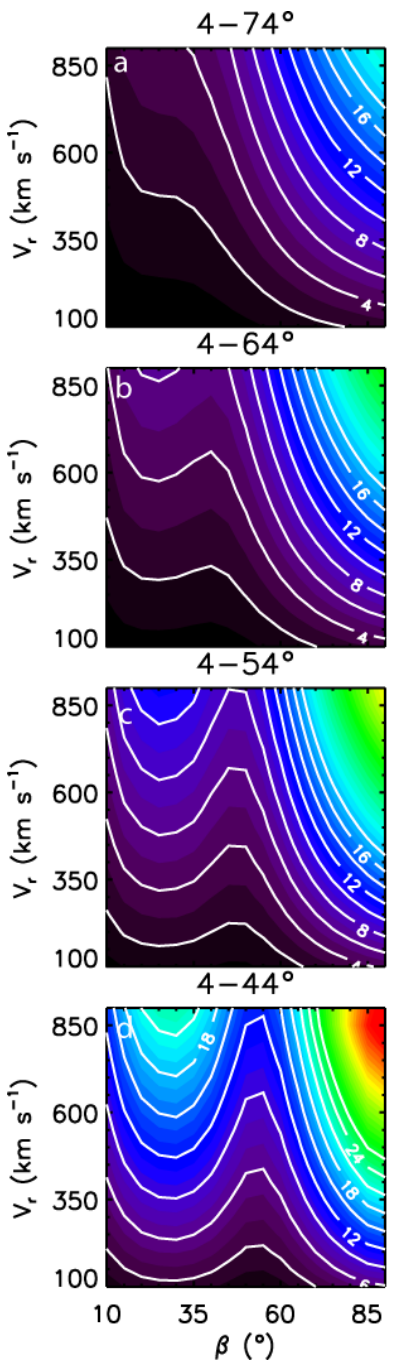

Fig. 6. Contour plots of the mean absolute error in $V_{r}$ as a function of $V_{r}$ and $\beta$. Each panel is derived from fitting tracks over the elongation range given. The mean absolute error is colour coded, with black (red) corresponding to small (large) values, and values are also marked on the overlaid contours. Note that the panels in the left hand column are on a common colour scale, and those on the right are on a common but different colour scale.

and higher, and another at mid-range value of $\beta, 30^{\circ}$ to $40^{\circ}$. It can be seen that one is normally more prominent than the other and the prominence of one error population over the other is dependent on the visible extent of the event.

Panel (a) of Fig. 6 shows the absolute mean error in $V_{r}$ when the fitting is done over the elongation variation of the full $\mathrm{HI}$ field of view, from $4^{\circ}$ to $74^{\circ}$, and has smaller values than any of the other panels. Here the smallest errors occur for $\beta=40^{\circ}$, while the largest errors are in the region where $\beta>=60^{\circ}$. Panels (b), (c) and (d) of Fig. 6 with maximum elongation extents of $44^{\circ}$ to $64^{\circ}$ show what errors you get when fitting out to approximately halfway through the HI-
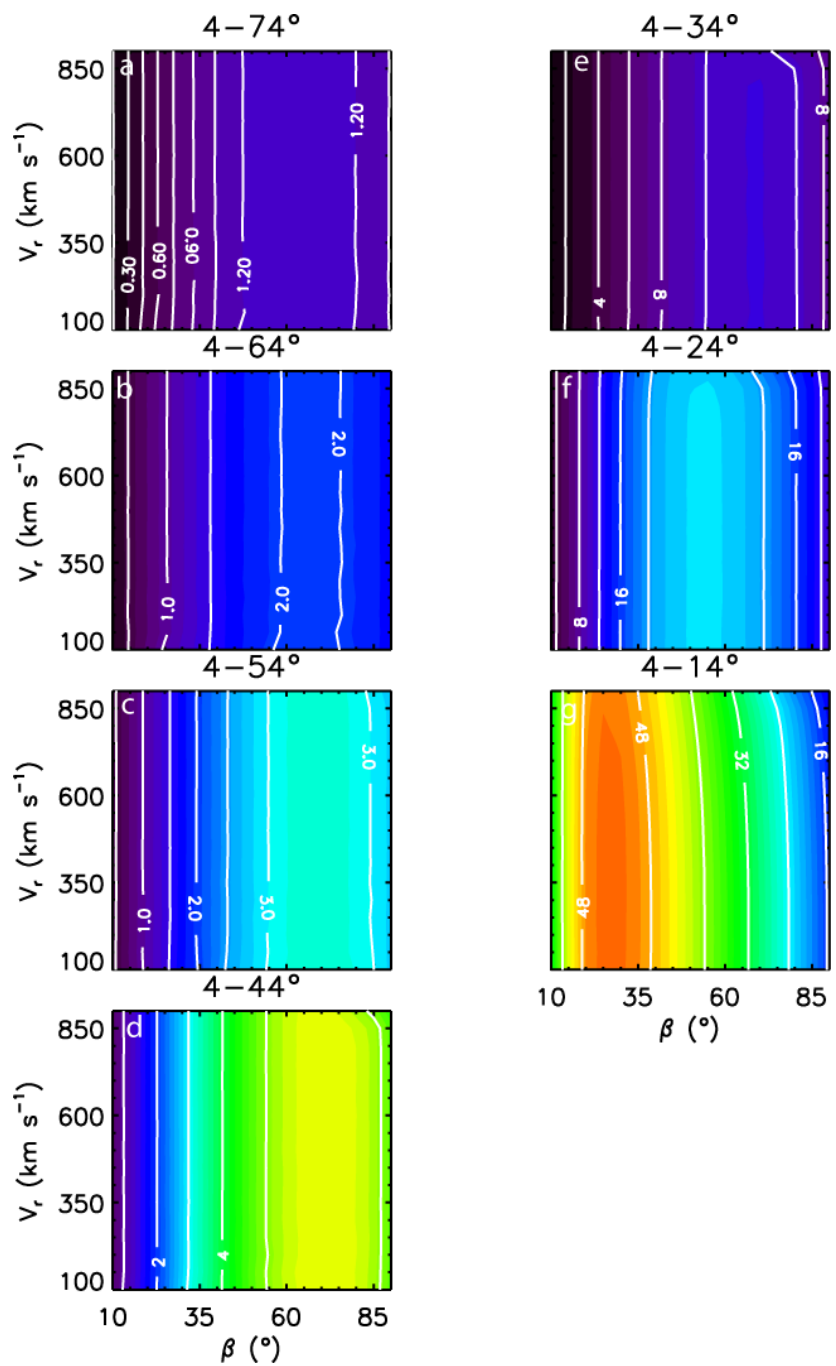

Fig. 7. Contour plots of the mean absolute error in $\beta$ as a function of $V_{r}$ and $\beta$. Each panel is derived from fitting tracks over the elongation range given. The mean absolute error is colour coded, with black (red) corresponding to small (large) values, and values are also marked on the overlaid contours. Note that the panels in the left hand column are on a common colour scale, and those on the right are on a common but different colour scale.

2 field-of-view. The errors here are of the similar scale but slightly larger than that in panel (a), but the location of minimum error has shifted to the region of $\beta=50^{\circ}$ to $\beta=60^{\circ}$, while a second region of maximum error forms at lower values of $\beta$ as previously mentioned. Clearly this shows that the method works best when an event is seen throughout the elongation range of the HI-1 and HI-2 instruments.

Panels (e), (f) and (g) of Fig. 6, where fitting is done to a maximum elongation of $34^{\circ}$, show much larger errors than panels $(\mathrm{a}-\mathrm{d})$. Here, the error is at a minimum in the region of $\beta=60^{\circ}$ to $\beta=70^{\circ}$ and a maximum in the region $\beta=30^{\circ}$ to $\beta=40$, while the error population at larger $\beta$ values 
disappears entirely. Clearly this shows that the important part of the fit for smaller values of $\beta$ occur at larger elongations, whereas for larger values of $\beta$ the early region of the fit describes the event quite well. Again note the increasing size of the errors with diminishing elongation extent.

Figure 7, like Fig. 6, shows the mean absolute error in $\beta$ as function of the input $V_{r}$ and $\beta$ for fits to the same ranges of elongations as shown in Figs. 5 and 6. Again the colour scale is different in the panels $(\mathrm{e}-\mathrm{g})$ to what is used in panels ( $\mathrm{a}-$ d), increasing in maximum value as the extent of elongation used in the fit decreases; again the overlaid contours show this. Here, similar to the case for $V_{r}$, the smallest errors occur when $\beta$ is the smallest and, much like the case of $V_{r}$, this is true for any given velocity. Unlike the error in $V_{r}$ all panels have a similar pattern of near uniform increases in error in $\beta$ with increasing $\beta$.

While the mean absolute error behaves in a similar manner in each panel, the elongation range over which the fit is performed has a much larger effect on the size of the mean absolute error. At large elongation ranges, as in panels (a-d), the values for the mean fit error are much lower across the range of $V_{r}$ and $\beta$, while in the elongation range used in panels (f) and (g) $\left(4^{\circ}\right.$ to $24^{\circ}, 4^{\circ}$ to $\left.14^{\circ}\right)$ the mean fit error is much larger. Compare the maximum errors, $\approx 1.5^{\circ}$ in the elongation range $4^{\circ}$ to $74^{\circ}$ with $\approx 25^{\circ}$ in the elongation range $4^{\circ}$ to $24^{\circ}$. This large difference makes accurately identifying the direction of transient events much more difficult when only using the HI-1 instrument.

\section{Discussion}

We have investigated the accuracy of determining the velocity and direction of solar wind transients observed by the STEREO HI instrument. As has been shown, events which are not seen out to a large elongation have a less accurate fit, such as events which are faint in intensity. One factor that will influence the elongation out to which a solar transient can be observed is its direction of propagation relative to the so-called Thomson Sphere (e.g. Vourlidas and Howard, 2006). The Thomson sphere, the surface from which the HI cameras will see the most scatter of light from electrons, is a sphere in which the Sun-Spacecraft line forms a diameter. Solar transients propagating at a large $\beta$ will move outside the Thomson Sphere at shorter distances from the Sun and this will tend to hinder their visibility to large elongations. Obviously, despite this effect transients with large associated densities, such as those likely to create adverse conditions on Earth will still be seen out to over $40^{\circ}$ elongation. Clearly Earth-bound events, which could have detrimental effects on satellite in orbit around the Earth, can be seen and predicted best while the spacecraft are within $40-50^{\circ}$ of the Earth. The combinations of this technique with the images used to make up a J-map allow for a good estimate of speed, direction and potentially geo-effectiveness when applied to Earth directed events.

The Thomson Sphere is also going to reduce the visibility of most events that do not pass close to the spacecraft at large elongations. In addition Thomson Sphere effects can change what part of a transient is imaged as it evolves in shape and structure throughout the heliosphere. This effect for wide CMEs, where the leading edge is not necessarily the same as the observed leading edge, has been noted by Webb et al. (2009) and Lugaz et al. (2009). If it is the case that different parts of a CME front are observed with time it can result in a slightly varying value for $\beta$ and will obviously result in a different profile in a J-map which is clearly a source of additional error to be aware of when using this technique. However, analysis of planetary-bound events has been undertaken with success, through verification by in-situ measurements, at both Venus (Rouillard et al., 2009b) and Earth (Rouillard et al., 2009a, c, for example) to track a variety of transient events.

It has been shown that the extent to which an event can be seen in the HI cameras has a significant effect on the accuracy of the fit. Indeed the difficulty associated with being sure of the fit has been noted by Savani et al. (2009), where they state that only including data from the HI-1 camera introduces large uncertainties in the fitting results. While this is true for events with a small $\beta$, we have shown that for larger $\beta$ values the error in $V_{r}$ is approximately the same regardless of the extent to which the event is seen. Obviously the only way to measure the true velocity of an event is through the use of in-situ data and this will only be possible for events that pass over a spacecraft with the tools to measure the plasma properties. As mentioned previously the analysis method used here assumes discrete plasma points and makes no measurement of the angular size of the event in the ecliptic plane, although this can be estimated if the event can be seen in both STEREO spacecraft. In this case each STEREO spacecraft will image different parts of the same event and so an approximation of the angular size can be made.

The analysis assumes that the event velocity is constant neglecting any acceleration phase that may occur near the sun. It is assumed that any acceleration occurs before the event enters the field of view. Generally transient events tend to accelerate or decelerate towards the ambient solar wind speed, as shown by Gopalswamy et al. (2000) and Jones et al. (2007) for example; in addition there is evidence of cases of "late accelerating" transient events as reported by Tappin (2006). In both these cases the profile of the transient in a J-map will be altered. These changes in the profile on the J-map will not necessarily be apparent to an observer resulting in an additional error in the estimates of $\mathrm{V}_{r}$ and $\beta$. As the profile of an accelerating or decelerating transient event is not governed by Eq. (1) the estimates of $V_{r}$ and $\beta$ will have a greater uncertainty. The start of the field of view at $4^{\circ}$ elongation corresponds to a minimum distance of $16 R_{\text {sun }}$, 
although this value is dependent on the angle of propagation of the event. For larger $\beta$ values the minimum distance from the Sun to the field of view of the HI cameras is smallest (see Fig. 1). This makes the assumption of constant velocity in the HI field of view less likely to be valid for such events. As noted by Savani et al. (2009) and Sheeley et al. (1999), acceleration has generally ceased before the event reaches the C3 coronagraph of the LASCO instrument at a height of approximately $30 R_{\text {sun }}$. Conversely for events with a small $\beta$ the minimum distance between the Sun and the HI field of view is larger and so the assumption of constant velocity is more likely to be valid provided that the transient velocity is not significantly larger than the ambient solar wind speed when deceleration effects may play an important role. Interestingly when an event is only seen out to small elongations the area with the smallest error in velocity is that of high $\beta$ and low velocity. As these are the most likely to still be in the acceleration phase this could be seen as a possible false result and events which fall into this category should be examined carefully. This combined with the relatively high errors associated with high values of $\beta$ due to the fitting method makes these events harder to accurately parameterize. The additional errors associated with the assumptions of the method as described could result in an error on the values of $V_{r}$ and $\beta$ that is comparable to the error examined here and users should be aware of this when applying this method to the analysis of transient events using J-maps.

The method examined here uses observations from only one of the STEREO spacecraft; from this a direction can be obtained for the propagation of transient events, although an elongation range extending over $40^{\circ}$ is required for an accurate fit. Although observations from one spacecraft are insufficient to determine the longitudinal extent of a transient event, this method still allows for an estimate of the direction which can then be used to estimate if the event will impact on the near-Earth environment. Given STEREOs capability to observe an event from multiple angles and so estimate the longitudinal size it should then be possible to improve the estimate on the likelihood of impact on the near-Earth environment. Other methods which make use of CME models and observations from the coronagraphs on the STEREO spacecraft such as by Thernisien et al. (2009), de Koning et al. (2009) and Maloney et al. (2009) can also provide an estimate of the longitudinal size of a transient and the J-plot technique could complement the work done by these authors.

\section{Conclusions}

The HI cameras on the STEREO spacecraft have the potential to be a powerful predictive tool for Space Weather. We have shown that the manual selection of points must be done with care to ensure the best fit to an elongation profile for any given transient event. We have also shown how the extent in the $\mathrm{HI}$ instruments to which a transient event is seen affects the accuracy of the resulting fit. This allows for the velocity and direction of an event to be stated with a statistical confidence limit for the effect of the method examined here. Finally we demonstrated how the mean fit error varies for different values of $V_{r}$ and $\beta$, finding that, in general, the error in $V_{r}$ increases with velocity for any given $\beta$, although there are some differences when events are fitted out to different elongations. We also found that, in general, the error in $\beta$ increases with $\beta$ for any given $V_{r}$ and that unlike the error in $V_{r}$ the main difference here between events fitted out to different elongations was the size of the error. Hence we conclude that to accurately predict when Earth-bound events will arrive at $1 \mathrm{AU}$ the event must first be seen out to an elongation well into the HI-2 camera range. The beacon data sent from the STEREO spacecraft could be used to provide the range required and allow some warning for events which could strike the near-Earth environment. Ideally the event should be seen out to a minimum of $40^{\circ}$, although the further it can be seen the better the prediction of velocity and direction can be. Clearly if this is to be used in a space weather prediction context then a compromise must be reached between accuracy and allowing sufficient time to give a useful warning.

Acknowledgements. AOW is supported by a STFC CASE studentship held by the University of Leicester and the Rutherford Appleton Laboratory. The STEREO/SECCHI data are produced by a consortium of RAL (UK), NRL (USA), LMSAL (USA), GSFC (USA), MPS (Germany), CSL, (Belgium), IOTA (France) and IAS (France).

Topical Editor R. Forsyth thanks N. Lugaz and A. Breen for their help in evaluating this paper.

\section{References}

Baker, D., Rouillard, A. P., van Driel-Gesztelyi, L., Démoulin, P., Harra, L. K., Lavraud, B., Davies, J. A., Opitz, A., Luhmann, J. G., Sauvaud, J.-A., and Galvin, A. B.: Signatures of interchange reconnection: STEREO, ACE and Hinode observations combined, Ann. Geophys., 27, 3883-3897, 2009, http://www.ann-geophys.net/27/3883/2009/.

Brueckner, G. E., Howard, R. A., Koomen, M. J., Korendyke, C. M., Michels, D. J., Moses, J. D., Socker, D. G., Dere, K. P., Lamy, P. L., Llebaria, A., Bout, M. V., Schwenn, R., Simnett, G. M., Bedford, D. K., and Eyles, C. J.: The large angle spectroscopic coronagraph (LASCO), Solar Phys., 162(1-2), 357-402, 1995.

Davies, J. A., Harrison, R. A., Rouillard, A. P., Sheeley Jr., N. R., Perry, C. H., Bewsher, D., Davis, C. J., Eyles, C. J., Crothers, S. R., and Brown, D. S.: A synoptic view of solar transient evolution in the inner heliosphere using the Heliospheric Imagers on STEREO, Geophys. Res. Lett., 36, L02102, doi:10.1029/2008GL036182, 2009.

Davis, C. J., Davies, J. A., Lockwood, M., Rouillard, A. P., Eyles, C. J., and Harrison, R. A.: Stereoscopic imaging of an Earth-impacting solar coronal mass ejection: A major milestone for the STEREO mission, Geophys. Res. Lett., 36, L08102, doi:10.1029/2009GL038021, 2009. 
de Koning, C. A., Pizzo, V. J., and Biesecker, D. A.: Geometric localization of CMEs in 3D space using STEREO beacon data: First Results, Solar Phys., 256, 167-181, 2009.

Eyles, C. J., Harrison, R. A., Davis, C. J., Waltham, N. R., Shaughnessy, B. M., Mapson-Menard, H. C. A., Bewsher, D., Crothers, S. R., Davies, J. A., Simnett, G. M., Howard, R. A., Moses, J. D., Newmark, J. S., Socker, D. G., Halain, J., Defise, J., Mazy, E., and Rochis, P.: The Heliospheric Imagers Onboard the STEREO Mission, Solar Phys., 254(2), 387-445, 2009.

Eyles, C. J., Simnett, G. M., Cooke, M. P., Jackson, B. V., Buffington, A., Hick, P. P., Waltham, N. R., King, J. M., Anderson, P. A., and Holladay, P. E.: The solar mass ejection imager (SMEI), Solar Phys., 217(2), 319-347, 2003.

Gopalswamy, N., Lara, A., Lepping, R. P., Kaiser, M. L., Berdichevsky, D., and St Cyr, O. C.: Interplanetary acceleration of coronal mass ejections. Geophys. Res. Lett., 27, 145-148, 2000.

Howard, R. A., Moses, J. D., Vourlidas, A., Newmark, J. S., Socker, D. G., Plunkett, S. P., Korendyke, C. M., Cook, J. W., Hurley, A., Davila, J. M., Thompson, W. T., St Cyr, O. C., Mentzell, E., Mehalick, K., Lemen, J. R., Wuelser, J. P., Duncan, D. W., Tarbell, T. D., Wolfson, C. J., Moore, A., Harrison, R. A., Waltham, N. R., Lang, J., Davis, C. J., Eyles, C. J., Mapson-Menard, H., Simnett, G. M., Halain, J. P., Defise, J. M., Mazy, E., Rochus, P., Mercier, R., Ravet, M. F., Delmotte, F., Auchere, F., Delaboudinier, J. P., Bothmer, V., Deutsch, W., Wang, D., Rich, N., Cooper, S., Stephens, V., Maahs, G., Baugh, R., McMullin, D., and Carter, T.: Sun Earth Connection Coronal and Heliospheric Investigation (SECCHI), Space Sci. Rev., 136(1-4), 67$115,2008$.

Jones, R. A., Breen, A. R., Fallows, A. R., Canals, A., Bisi, M. M., and Lawrence, G.: Interaction between coronal mass ejections and the solar wind, J. Geophys. Res.-Space, 112, A08107, doi:10.1029/2006JA011875, 2007.

Lugaz, N., Vourlidas, A., Roussev, I. I., and Morgan, H.: SolarTerrestrial simulation in the STEREO era: The 24-25 January 2007 eruptions, Solar Phys., 256(1-2), 269-284, 2009.

Maloney, S. A., Gallagher, P. T., and McAteer, R. T. J.: Reconstructing the 3-D trajectories of CMEs in the inner heliosphere, Solar Phys. 256, 149-166, 2009.

Rouillard, A. P., Davies, J. A., Forsyth, R. J., Rees, A., Davis, C. J., Harrison, R. A., Lockwood, M., Bewsher, D., Crothers, S. R., Eyles, C. J., Hapgood, M., and Perry, C. H.: First imaging of corotating interaction regions using the STEREO spacecraft, Geophys. Res. Lett., 35(10), L10110, doi:10.1029/2008GL033767, 2008.

Rouillard, A. P., Davies, J. A., Lavraud, B., Forsyth, R. J., Savani, N. P., Bewsher, D., Brown, D. S., Sheeley, N. R., Davis, C. J., Harrison, R. A., Howard, R. A., Vourlidas, A., Lockwood, M., Crothers, S. R., and Eyles, C. J.: Intermittent release of small-scale transients in the slow solar wind: I, Remote sensing observations, J. Geophys. Res.-Space, accepted, doi:10.1029/2009JA014471, 2009a.

Rouillard, A. P., Davies, J. A., Forsyth, R. J., Savani, N. P., Sheeley, N. R., Thernisien, A., Zhang, T.-L., Howard, R. A., Anderson, B., Carr, C. M., Tsang, S., Lockwood, M., Davis, C. J., Harrison, R. A., Bewsher, D., Fränz, M., Crothers, S. R., Eyles, C. J., Brown, D. S., Whittaker, I., Hapgood, M., Coates, A. J., Jones, G. H., Grande, M., Frahm, R. A., and Winningham, J. D.: A solar storm observed from the Sun to Venus using the STEREO, Venus Express and MESSENGER spacecraft, J. Geophys. Res.-Space, 114, A07106, doi:10.1029/2008JA014034, 2009b.

Rouillard, A. P., Lavraud, B., Davies, J. A., Savani, N. P., Burlaga, L. F., Forsyth, R. J., Sauvaud, J. A., Opitz, A., Lockwood, M., Luhmann, J. G., Simunac, D. C., Galvin, A. B., Davis, C. J., and Harrison, R. A.: Intermittent release of small-scale transients in the slow solar wind: II In-situ evidence, J. Geophys. Res.-Space, accepted, doi:10.1029/2009JA014472, 2009c.

Rouillard, A. P., Savani, N. P., Davies, J. A., Lavraud, B., Forsyth, R. J., Morley, S. K., Opitz, A., Sheeley, N. R., Burlaga, L. F., Sauvaud, J., Simunac, K. D. C., Luhmann, J. G., Galvin, A. B., Crothers, S. R., Davis, C. J., Harrison, R. A., Lockwood, M., Eyles, C. J., Bewsher D., and Brown, D. S.: A Multispacecraft Analysis of a Small-Scale Transient Entrained by Solar Wind Streams, Solar Phys., 256(1-2), 307-326, 2009d.

Savani, N. P., Rouillard, A. P., Davies, J. A., Owens, M. J., Forsyth, R. J., Davis, C. J., and Harrison, R. A.: The radial width of a Coronal Mass Ejection between 0.1 and $0.4 \mathrm{AU}$ estimated from the Heliospheric Imager on STEREO. Ann. Geophys., accepted, 2009.

Sheeley, N. R., Walters, J. H., Wang, Y. M., and Howard, R. A.: Continuous tracking of coronal outflows: Two kinds of coronal mass ejections, J. Geophys. Res.-Space, 104(A11), 2473924767, 1999.

Sheeley Jr., N. R., Herbst, A. D., Palatchi, C. A., Wang, Y., Howard, R. A., Moses, J. D., Vourlidas, A., Newmark, J. S., Socker, D. G., Plunkett, S. P., Korendyke, C. M., Burlaga, L. F., Davila, J. M., Thompson, W. T., St Cyr, O. C., Harrison, R. A., Davis, C. J., Eyles, C. J., Halain, J. P., Wang, D., Rich, N. B., Battams, K., Esfandiari, E., and Stenborg, G.: Secchi observations of the Sun's garden-hose density spiral, Astrophys. Lett., 674(2), L109-L112, 2008a.

Sheeley, N. R., Herbst, A. D., Palatchi, C. A., Wang, Y., Howard, R. A., Moses, J. D., Vourlidas, A., Newmark, J. S., Socker, D. G., Plunkett, S. P., Korendyke, C. M., Burlaga, L. F., Davila, J. M., Thompson, W. T., St Cyr, O. C., Harrison, R. A., Davis, C. J., Eyles, C. J., Halain, J. P., Wang, D., Rich, N. B., Battams, K., Esfandiari, E., and Stenborg, G.: Heliospheric images of the solar wind at Earth, Astrophys. J., 675(1), 853-862, 2008b.

Tappin, S. J.: The deceleration of an interplanetary transient from the sun to 5 AU, Solar Phys., 233, 233-248, 2006.

Thernisien, A., Vourlidas, A., and Howard, R. A.: Forward modeling of Coronal Mass Ejections using STEREO/SECCHI Data, Solar Phys., 256, 111-130, 2009.

Vourlidas, A. and Howard, R. A.: The proper treatment of coronal mass ejection brightness: A new methodology and implications for observations, Astrophys. J., 642(2), 1216-1221, 2006.

Webb, D. F., Howard, T. A., Fry, C. D., Kuchar, T. A., Odstrcil, D., Jackson, B. V., Bisi, M. M., Harrison, R. A., Morrill, J. S., Howard, R. A., and Johnstone, J. C.: Study of CME propagation in the inner heliosphere: SOHO LASCO, SMEI and STEREO HI observations of the January 2007 events, Solar Phys., 256(1-2), 239-267, 2009. 